\title{
Basic technology and proper usage of home health monitoring devices
}

\author{
Himel Mondal, Shaikat Mondal
}

Mondal H, Mondal S. Basic technology and proper usage of home health monitoring devices. Malays Fam Physician. 2021;16(1);8-14. https://doi.org/10.51866/rv1097

\section{Keywords:}

COVID-19, Blood Glucose,

Blood Pressure Monitors,

Primary Health Care, Remote

Consultation

\section{Authors:}

\section{Himel Mondal}

(Corresponding author)

MBBS, MD

Department of Physiology

Bhima Bhoi Medical College and Hospital Balangir, Odisha, India.

Email: himelmkcg@gmail.com

\section{Shaikat Mondal}

MBBS, MD

Department of Physiology

Raiganj Government Medical College and Hospital, West Bengal, India.

\section{Abstract}

Home health monitoring devices are consumer-grade devices that help to monitor the health of individuals at home. These devices are usually low-cost and easily procurable, and they can be operated by patients or their caretakers with minimal training. However, improper usage of these devices may provide erroneous results, which can lead to an unnecessary hospital visit or teleconsultation. In this article, we discuss the basic technology and proper usage of some of these devices, namely automatic blood pressure monitors, blood glucose monitors, body fat monitors, pulse oximeters, electrocardiographs, digital thermometers, and infrared thermometers. This brief document intends to help primary health care professionals and their patients use these devices.

\section{Introduction}

Home health monitoring devices are consumer-grade devices that help to monitor and track the health of individuals outside of clinics. ${ }^{1}$ Patients or their caretakers can monitor health status from their homes with these devices. ${ }^{2}$ The costs of these devices are usually lower than those of hospital-grade devices, and home devices can be operated easily by patients. ${ }^{3}$ Measured parameters can be recorded for self-monitoring purposes or consultation with doctors. ${ }^{4}$ Proper usage of these devices is imperative for credible results. Faulty handling of home devices or improper self-preparation may lead to erroneous results. If patients or their caretakers do not follow proper measurement techniques, 5,6 they could make unnecessary visits to medical facilities or consult superfluously with doctors via telemedicine channels. In this article, we present the basic technology (i.e., fundamental principles upon which the equipment works) and proper usage of some home health monitoring devices, namely automatic blood pressure monitors, blood glucose monitors, body fat monitors, pulse oximeters, electrocardiographs, digital thermometers, and infrared thermometers.

\section{Automatic blood pressure monitor}

\section{Technology}

Upper arm and wrist type automatic blood pressure monitors are popular for monitoring blood pressure. ${ }^{7}$ Both of the devices use the oscillometric method to detect blood pressure. Figure 1a demonstrates the basic principles of blood pressure measurement. When the cuff pressure exceeds systolic blood pressure, the artery becomes occluded and blood does not flow below the cuff, thus generating no sound or vibrations [Figure 1a]. When the cuff pressure is reduced gradually and becomes lower than systolic pressure, turbulent blood flow occurs beyond the cuff, and Korotkoff sounds and vibrations form [Figure 1a]. This vibration is detected by the pressure sensor present in the automatic blood pressure monitor. When the cuff pressure sinks below the diastolic pressure, minimal vibrations and sounds are produced [Figure 1a]. ${ }^{8}$ Sensors in the monitor use a fixed algorithm to calculate the diastolic blood pressure from this point. Most blood pressure monitors have fully automated the inflation and deflation of the cuff. When the monitor inflates the cuff, "fuzzy logic" (logic in which values of variables may be any number between 0 and 1) is used to estimate systolic blood pressure. The monitor then inflates the cuff above this level and then deflates to measure blood pressure.' Although the underlying technologies are similar in devices manufactured by different companies, one must always ensure that individual devices have been approved by the appropriate authority (e.g., FDA, ISO). Furthermore, the device should be calibrated at frequent intervals. ${ }^{10}$ 


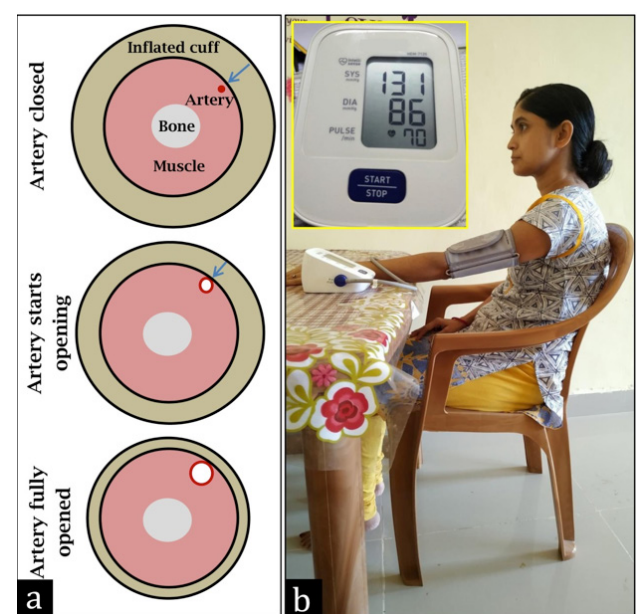

Figure 1. (a) Basic principle of oscillometric blood pressure measurement (b) A person measuring blood pressure using an automatic blood pressure monitor (inset: blood pressure reading)

\section{Usage}

Before measurement, the operator must ensure that the patient has avoided tea, coffee, caffeinated drinks, smoking, and any mode of exercise in the preceding 30 minutes. ${ }^{11,12}$ Any constricting clothes should be removed. After voiding the bladder, the patient sits quietly with uncrossed legs on a chair with back support and feet flat on the floor without shoes. The arm is supported on a flat surface, preferably on a table at the heart level. The patient rests for five minutes in this position. Nearby electronics gadgets should be kept away. The operator must then fasten the cuff on a bare arm and start recording the blood pressure [Figure $\mathbf{1 b}$ ]. The patient must not move or talk during the measurement. The operator measures twice with a gap of one minute in between, then takes the average of these measurements. ${ }^{13}$ The accuracy of this figure may be further enhanced by measuring thrice and taking the average as the final reading. ${ }^{14}$ However, these guidelines may vary among different countries. The final reading should always be noted in a logbook (i.e., a book where patients keep a record of their measurement) or health application in order to track changes over time. ${ }^{15}$

\section{Blood glucose monitor}

\section{Technology}

A blood glucose monitor and test strip are the basic components of a biosensor. Test strips contain a bioreceptor that receives a specific analyte and a transducer. Blood with various analytes [Figure 2a] comes into contact with the capillary space of the test strip. A bioreceptor in the strip specifically accepts glucose. This reaction generates a physiochemical signal that is converted to an electrical signal via a transducer and sent from the strip to the monitor. The monitor acts as the processing module and displays the result in a readable format. ${ }^{16}$ The monitor may also use predefined factors to convert the whole blood glucose reading to its plasma equivalent value (e.g., whole blood glucose $\times 1.11) .{ }^{17} \mathrm{~A}$ few monitors utilize a reaction in which glucose reacts with an enzyme and the product acts on a dye to change the color proportional to glucose concentration. ${ }^{18}$

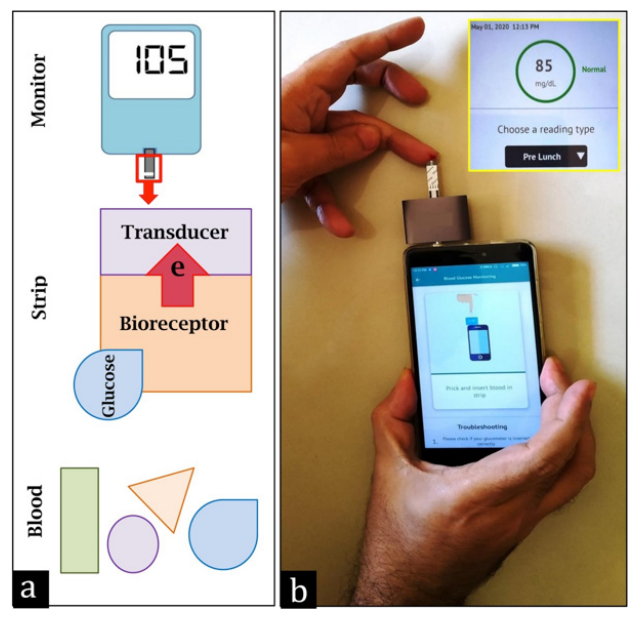

Figure 2. (a) Basic principle of a glucose monitor (b) A person measuring blood glucose on a smartphone-dependent glucose monitor (inset: blood sugar reading on a smartphone screen)

\section{Usage}

Operators should handle strips with dry and clean hands and keep the container of strips closed after extracting a single strip. Before measurement, checking the expiry date of the strip is mandatory. A new lancet should be used for each measurement. The lancet should be placed in the appropriate slot of the lancing device, and the depth of the prick should be set according to previous experience or skin thickness. The hand should be washed with mild soap and must be dry before pricking. After spring-loading the pricking device, a finger is pricked (preferably the ring or middle finger). Gentle milking may be necessary to produce an adequate drop of blood. The meter will take some time to perform the reaction after the blood makes contact with the strip capillary space. ${ }^{19}$ The reading will be displayed on the device screen or smartphone according to the type of device. Figure $\mathbf{2 b}$ illustrates the process 
of measuring blood glucose on a smartphonedependent glucose monitor by pricking the ring finger, and the result is shown in the inset. After the measurement, the lancet is disposed of in a sharp-materials container and strips in a biohazardous material container. Some patients may need to press the finger with cotton in order to stop the blood. After use, the tip of the lancet may be cleansed with an alcohol swab. The blood glucose reading should be entered into a logbook immediately after measurement.

\section{Body fat monitor}

\section{Technology}

Consumer-grade body fat monitors may come in either a two-electrode type without a weighing scale or a four-electrode type combined with a weighing scale. ${ }^{6}$ Figure 3a shows four electrodes placed on the hands and feet of a bioelectrical impedance analysis body fat monitor. The body fat monitor uses a low-frequency current that passes through the body easily via the watery medium and is impeded by fat. One electrode sends the current and another electrode receives the signal. This principle is used to estimate total body water and fat-free mass. Fat-free mass is subtracted from the measured body weight to calculate fat mass. ${ }^{20}$ This calculation is programmed into the monitor software and varies between different human ethnicities. Thus, it is important to check if individual monitors have been recommended by a regional licensing authority. ${ }^{21}$ In two-electrode body fat monitors (either hands or feet), the weight of the person is manually entered. By comparison, four-electrode body fat monitors simultaneously measure and provide body fat readings.

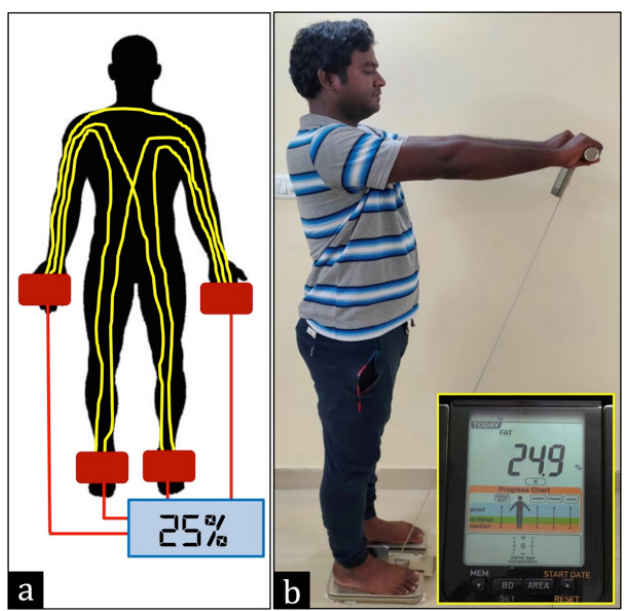

Figure 3. (a) Basic principle of bioelectric impedance analysis (b) A person measuring body fat on a four-electrode monitor (inset: body fat percentage reading)

\section{Usage}

Random measurement of body fat may lead to erroneous results. Patients should take premeasurement precautions when measuring body fat, including refraining from alcohol for 48 hours; avoiding coffee, chocolate, or any other diuretics for 12 hours; and abstaining from food and drink for four hours. Exercise should be avoided for 12 hours. Measurement during any illness that may affect body hydration such as fever and diarrhea should be avoided. The bladder should be voided within 30 minutes of measurement. During measurement, precise demographic data such as age, sex, and height should be entered on the monitor. ${ }^{22,23}$ All of these are used in the prediction equation. Proper contact with electrodes and proper body position should be maintained [Figure $\mathbf{3 b}$ ]. If hands and feet are extremely dry, these should be moistened with a wet handkerchief. Movement during measurement should be avoided. Commonly, body fat is expressed as a percentage [Figure $\mathbf{3 b}$ inset]. However, a single measurement should not be taken as final; instead, the average of three consecutive measurements should be calculated and recorded in a logbook or heath application.

\section{Electrocardiograph}

\section{Technology}

Single-lead consumer-grade electrocardiographs are now available at affordable prices. These devices work on the same principles as hospitalgrade electrocardiographs. The electrical activity of the heart is conducted to the body surface and captured by the electrodes placed on the skin [Figure 4a]. As this signal is very weak, the device amplifies it, and a simple galvanometer detects changes in voltages. This signal is processed by a computer, and the wave pattern is either shown on the device screen or on a connected smartphone [Figure $\mathbf{4 b}$ inset]. The computer also analyses the rate and rhythm of the heartbeats to detect any gross abnormalities. ${ }^{24,25}$ Typically, these devices have two electrodes placed on the surface of the casing. These two electrodes are either touched by two hands or touched by the right hand and the left side of the abdomen [Figure $\mathbf{4 b}$ ]. 


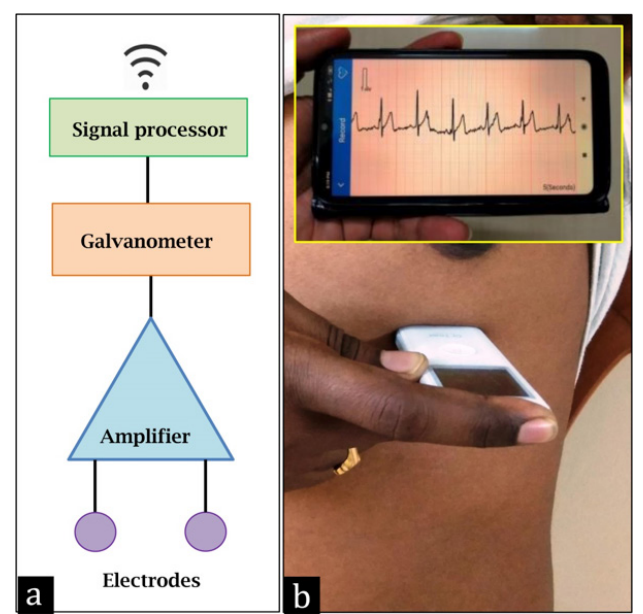

Figure 4. (a) Basic principle of an electrocardiograph (b) A person measuring a single-lead electrocardiogram using a consumer-grade electrocardiograph (inset: an electrocardiogram on a smartphone screen)

\section{Usage}

Electronic gadgets, metallic substances, and magnetic materials should be kept away from the device during measurement. A five-minute rest in a quiet sitting position is recommended before measurement. The person being measured should not move or talk during measurement. Optimum contact with the electrodes of the device should be ensured; if the hands are dry, moisten them by wiping them with a wet handkerchief. According to the manufacturer's instructions, place the electrodes properly to obtain credible ECG tracing (e.g., in Figure $\mathbf{4 b}$, the subject is holding the device with one electrode on the right index finger and another electrode on the left abdomen for a typical lead II measurement). After measurement, the report should be reviewed by a doctor. $^{26}$

\section{Pulse oximeter}

\section{Technology}

Pulse oximeters are commonly used in critical care settings and in anesthesia for monitoring the oxygen saturation of blood. However, handheld devices are available for use at home. These devices are battery-operated, standalone devices that can show pulse rates and oxygen saturation. The probe combines two light-emitting diodes (LED), red LED and infrared LED, which emit red and infrared light. The infrared light is not visible. Thus, we can only see red light from one side of the probe. On the opposite side of the probe, there is a light detector [Figure 5a]. When red and infrared light waves pass through the finger, some light is absorbed by the tissue and some is absorbed by the pulsating blood. The sensor removes the static signal (absorbed by the tissue) and only takes pulsatile signals. Oxyhemoglobin and deoxyhemoglobin display different patterns of absorption of red and infrared light. This absorption pattern is analyzed via the signal processing unit, and the result is displayed in a readable form. ${ }^{27,28}$

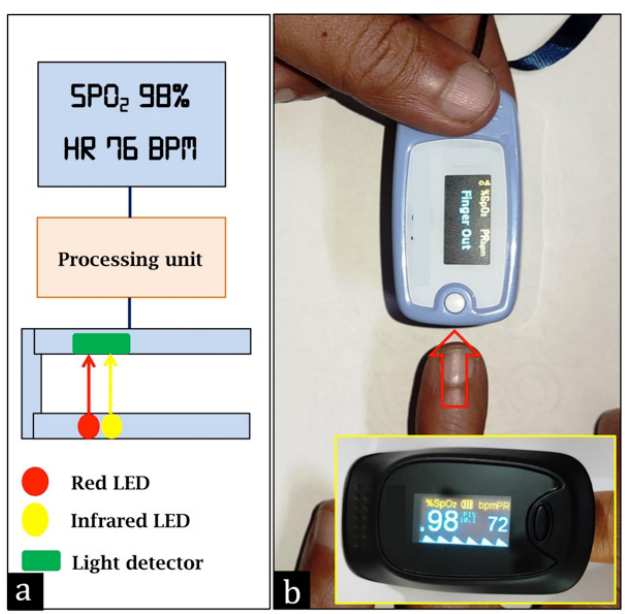

Figure 5. (a) Basic principle of a pulse oximeter (b) A person inserting finger into the pulse oximeter probe (inset: oxygen saturation and pulse rate reading)

\section{Usage}

Visible dirty hands should be washed, and tattooed or colored fingers (e.g., temporary henna designs) should be avoided. Nail color may be removed on the specific finger used for measurement. The probe should be placed on an appropriate finger so that it is neither too loose nor too tight. Figure $\mathbf{5 b}$ shows a person inserting a finger into the pulse oximeter probe. Care should be taken to check if the finger is covering the LEDs and the sensor on the opposite side. Pulse oximeters should not be used under bright sunlight or lighting, as interference from bright light may result in errors in estimation. If bright light cannot be avoided, a cloth can be used to cover the oximeter and finger. The person being measured should not move the finger during measurement. After using the device for several days, the LED and sensors can be cleaned with an alcohol swab as dirt may impair results. ${ }^{29}$

\section{Digital thermometer}

\section{Technology}

The digital thermometer uses the property of a thermistor (i.e., heating decreases resistance and 
cooling increases it). The thermometer has a tiny battery that sends a current through a wire towards the tip where the thermistor is present [Figure 6a]. According to the temperature of the thermistor, the current is impeded and received on the other end of the processing unit. This signal is then processed and the temperature is shown on the screen. ${ }^{30}$

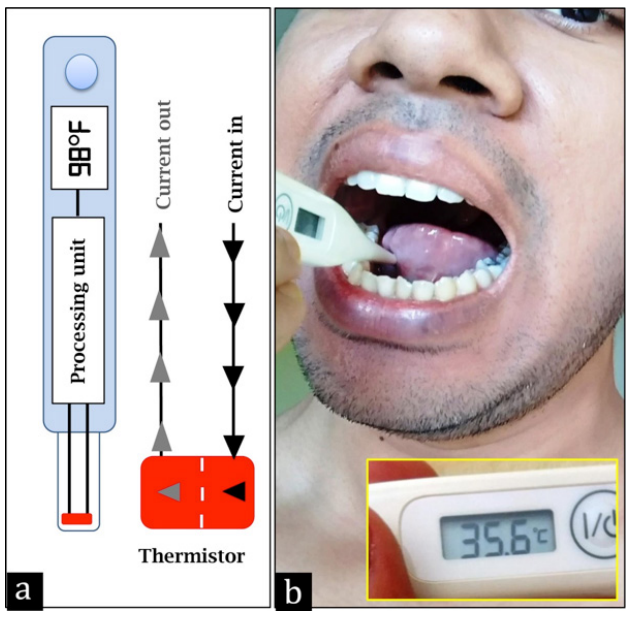

Figure 6. (a) Basic principle of a digital thermometer (b) A person placing the thermometer tip under the tongue (inset: temperature reading)

\section{Usage}

Digital thermometers can be used to safely record the temperature of all common sitesunder the tongue, armpit, and rectum. The thermometer should be handled with clean washed hands. The thermometer should also be cleaned with an alcohol swab or with soap and water after use. However, care should be taken not to dip the thermometer into water or liquid, which may damage the circuit inside. When measuring oral temperature, a person should not eat or drink within 30 minutes of measurement. After switching on the thermometer, it should be kept under the tongue [Figure $\mathbf{6 b}$ ], and the user should close his or her mouth firmly. The majority of thermometers beep after the measurement is recorded. Thus, wait until a beep sounds. The temperature should be immediately recorded in a logbook. For armpit measurement, the thermometer tip should come into contact with the skin. For rectal temperature measurement, the thermometer used should be designated specifically for the measurement of rectal temperature, and strict disinfection procedures should be followed. ${ }^{31}$

\section{Infrared thermometer}

\section{Technology}

Non-contact infrared thermometers can be used from a distance to measure temperature. These devices are larger in size than digital thermometers. The working principle is also different from that of a digital thermometer. Infrared thermometers receive infrared light emitted from a subject or an object-the warmer the subject or object, the greater its emissions of infrared radiation. These light rays are condensed via a lens in the device that focuses it on a thermopile (i.e., the sensor). The thermopile converts the infrared to heat, and the heat is converted to electricity using a processor. Finally, the electricity is processed and the reading is displayed [Figure 7a] ${ }^{32}$ Noncontact thermometers are often equipped with laser light. This is used to aim the thermometer at the desired point of interest. In Figure $\mathbf{7 b}$, the laser is seen on the middle of the forehead. This is simply for aiming the thermometer and has no connection to the heat sensing technology. Thus, many non-contact thermometers do not involve laser light. Both physicians and patients must be careful when selecting thermometers for measuring body temperature. Many available thermometers are for industrial usage. Care must be taken to confirm the temperature ranges that can be measured by the device as well as the device's certification according to local guidelines (e.g., FDA, ISO).

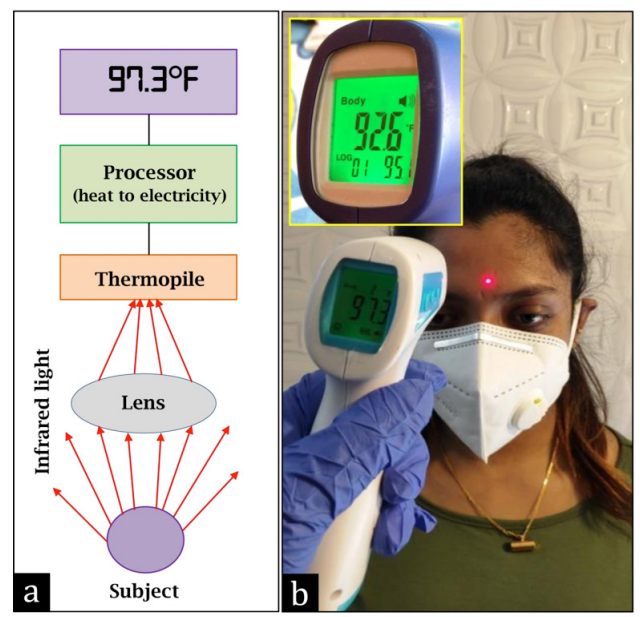

Figure 7. (a) Basic principle of an infrared thermometer (b) A person pointing the thermometer at the forehead of a subject from a distance (inset: temperature reading) 


\section{Usage}

The usage of non-contact thermometers has increased throughout the current COVID-19 pandemic. These devices are not only used in healthcare facilities but are being used in other non-healthcare facilities such as airports, shopping malls, banks, and so on. The device can also be used by family members to measure temperature from a distance when any one of the family members is in home quarantine. Care must be taken to obtain the proper temperature. The area of interest (e.g., forehead) should be cleaned and made dry before measurement. However, cosmetic wipes should be avoided, as these may increase the recorded temperature. Any additional clothing on the head should be removed, as this may increase the forehead temperature as well. The subject should not stand or sit under direct sunlight or near any heat source. The temperature of the environment should be between 16 to $40^{\circ} \mathrm{C}$, and the humidity should be below $85 \%$. The thermometer should be kept in the environment of measurement for 10 to 30 minutes so that the device adjusts to the ambient temperature. After taking these precautions, the thermometer must be held with the sensing part perpendicular to the measurement site, and the device must be switched on. ${ }^{33}$ Typically, it takes one to two seconds for the reading to show. These devices are easy to use and, being non-contact, help to prevent diseases that spread via contaminated surfaces. The lens of the device should be kept clean with a clean cloth, and the surfaces may be disinfected with a swab. However, the device should not be put into any disinfectant fluid.

\section{Conclusion}

This article compiled brief information on the technology and proper usage of the six most commonly used home health monitoring devices_automatic blood pressure monitors, blood glucose monitors, body fat monitors, pulse oximeter, electrocardiographs, digital thermometers, and infrared thermometers. This would help a busy physician to understand the basic working principles of the devices at a glance. However, this is a very basic guide. For an in-depth understanding, one may refer to the literature cited in this article. This guide would also help to disseminate knowledge amongst patients on the proper usage of the devices.

\section{Acknowledgments}

We thank the Head of the Department of Physiology, the Head of the Department of Anaesthesia, Dr. Sairavi Kiran Biri, Dr. Debasish Das, Dr. Dhananjay Soren of Fakir Mohan Medical College, Balasore, Odisha, Mr. Abhijit Roy of Roy Tech Tips, Ms. Smita Biswas of Smita's Boutique, and Ms. Shireen Sheikh, Barasat, West Bengal, India for their encouragement and help in the preparation of this manuscript. We also thank all the participants who volunteered as models in the figures.

\section{Funding}

No funding received for this work

\section{Ethical clearance}

Ethical clearance is not required

\section{Conflict of interest}

All authors declared there were no conflicts of interest

\section{How does this paper make a difference to general practice?}

- It informs primary care physicians on the working principles and correct handling of common home health monitoring devices.

- These physicians will then be able to train their patients to perform proper measurements.

- This guide can be shared with patients as well for the sake of their education.

- During or after a pandemic, when visiting a clinic is difficult, proper usage of home health monitoring devices can help patients and practitioners of telemedicine. 


\section{References}

1. Home Health Monitoring Devices. Somnology. 2016 July 26. Accessed 09 July 2020 at https:// www.somnologymd.com/2016/07/homehealth-monitoring-devices.

2. Story MF. Medical Devices in Home Health Care. National Academies Press (US). 2010. Accessed 09 July 2020 at https://www.ncbi. nlm.nih.gov/books/NBK210047.

3. Dias D, Paulo Silva Cunha J. Wearable Health Devices-Vital Sign Monitoring, Systems and Technologies. Sensors (Basel) 2018;18(8):2414.

4. Togawa T. Home health monitoring. J Med Dent Sci 1998;45(3):151-60.

5. Mondal S, Mondal H, Dutta R, Pal A, Acharya SS, Baidya C. Competency in Home Blood Pressure Monitoring and Effect of Training Program on Competency. Journal of Clinical and Diagnostic Research 2018;12:OC10-OC14.

6. Mondal H, Mondal S, Baidya C. Competency in home body fat monitoring by portable devices based on bioelectrical impedance analysis: A pilot study. J Educ Health Promot 2019;8:223.

7. Beevers G, Lip GY, O’Brien E. ABC of hypertension. Blood pressure measurement. Part I-sphygmomanometry: Factors common to all techniques. BMJ. 2001;322(7292):981-985.

8. Berger A. Oscillatory Blood

Pressure Monitoring Devices. BMJ 2001;323(7318):919.

9. Lin CT, Liu SH, Wang JJ, Wen ZC. Reduction of interference in oscillometric arterial blood pressure measurement using fuzzy logic. IEEE Trans Biomed Eng 2003;50(4):432-41.

10. Calibration, Maintenance, and Use of Blood Pressure Devices. National High Blood Pressure Education Program. 2004 August. Accessed on 09 July 2020 at https://www.ncbi.nlm.nih.gov/ books/NBK9622.

11. Ogedegbe G, Pickering T. Principles and techniques of blood pressure measurement. Cardiol Clin. 2010;28(4):571-586.

12. George J, MacDonald T. Home Blood Pressure Monitoring. Eur Cardiol. 2015;10(2):95-101.
13. Things you need to know about blood pressure and hypertension. Can J Cardiol. 2006;22(7):601-602.

14. Jose AP, Awasthi A, Kondal D, Kapoor M, Roy A, Prabhakaran D. Impact of repeated blood pressure measurement on blood pressure categorization in a population-based study from India. J Hum Hypertens 2019;33:594-601.

15. Monitoring Your Blood Pressure at Home [Internet]. USA: American Heart Association; 2017. Available from: https://www.heart. org/en/health-topics/high-blood-pressure/ understanding-blood-pressure-readings/ monitoring-your-blood-pressure-at-home. Accessed on 28 April 2020.

16. Wang J. Electrochemical glucose biosensors. Chem Rev 2008;108:814-25.

17. Kim HS. Blood Glucose Measurement: Is Serum Equal to Plasma?. Diabetes Metab J 2016;40(5):365-6.

18. Villena Gonzales W, Mobashsher AT, Abbosh A. The Progress of Glucose Monitoring-A Review of Invasive to Minimally and NonInvasive Techniques, Devices and Sensors. Sensors (Basel) 2019;19(4):800

19. Kirk JK, Stegner J. Self-monitoring of blood glucose: practical aspects. J Diabetes Sci Technol 2010;4:435-9.

20. Kyle UG, Bosaeus I, De Lorenzo AD, Deurenberg P, Elia M, Gómez JM, et al. Bioelectrical impedance analysis--part I: review of principles and methods. Clin Nutr 2004;23(5):1226-43.

21. Duren DL, Sherwood RJ, Czerwinski SA, et al. Body composition methods: comparisons and interpretation. J Diabetes Sci Technol 2008;2(6):1139-46.

22. Ricciardi R, Talbot LA. Use of bioelectrical impedance analysis in the evaluation, treatment, and prevention of overweight and obesity. J Am Acad Nurse Pract 2007;19:235-41.

23. Guedes DP. Clinical procedures used for analysis of the body composition. Rev Bras Cineantropom Desempenho Hum 2013;15:113-29.
24. Bansal A, Joshi R. Portable out-of-hospital electrocardiography: A review of current technologies. J Arrhythm 2018;34(2):129-38.

25. Garvey JL. ECG techniques and technologies. Emerg Med Clin North Am 2006;24(1):209-25.

26. AliveCor Heart Monitor and AliveECG app (Kardia Mobile) for detecting atrial fibrillation [Internet]. UK: National Institute for Health and Care Excellence; 2015. Available from: https://www.nice.org.uk/advice/mib35/chapter/ technology-overview. Accessed 28 April 2020.

27. Jubran A. Pulse oximetry. Crit Care 2015;19(1):272.

28. Singh S, Khan SZ, Singh D, Verma S, Talwar A. The uses of overnight pulse oximetry. Lung India 2020;37(2):151-7.

29. Using the Pulse Oximeter [Internet]. Switzerland: World Health Organization; 2011. Available from: https:/www.who.int/ patientsafety/safesurgery/pulse_oximetry/ who_ps_pulse_oxymetry_tutorial2_advanced_ en.pdf. Accessed 28 April 2020.

30. Gallagher JE. Inspection, Testing, Verification, Calibration, and Certification. In: Inspection, Testing, Verification, Calibration, and Certification. USA: Gulf Publishing Company; 2006.

31. Thermometers: How to Take your Temperature [Internet]. USA: Cleveland Clinic; 2020. Available from: https://my.clevelandclinic.org/ health/articles/9959-thermometers-how-totake-your-temperature. Accessed 28 April 2020.

32. How do infrared thermometers work? Generaltools. 2016 February 15. Accessed on 09 July 2020 at https://www.generaltools.com/ blog/how-do-infrared-thermometers-work.

33. Non-contact Infrared Thermometers. U.S. Food and Drug. 2020 April 23. Accessed on 09 July 2020 at https://www.fda.gov/medicaldevices/general-hospital-devices-and-supplies/ non-contact-infrared-thermometers.

34. Infrared thermometers: Cleaning and Storing. Temperature Blog. 2018 August 17. Accessed on 09 July 2020 at https://temperature.co.uk/ infrared-thermometers-cleaning-storing. 